ARTICLE

Received 20 Sep 2013 | Accepted 5 Feb 2014 | Published 6 Mar 2014 DOI: 10.1038/ncomms4394

\title{
Tousled-like kinases phosphorylate Asf1 to promote histone supply during DNA replication
}

IInaz M. Klimovskaia ${ }^{1, \dagger}$, Clifford Young ${ }^{2,3}$, Caroline B. Strømme ${ }^{1}$, Patrice Menard ${ }^{1, \dagger}$, Zuzana Jasencakova ${ }^{1}$, Jakob Mejlvang ${ }^{1, \dagger}$, Katrine Ask ${ }^{1}$, Michael Ploug ${ }^{4}$, Michael L. Nielsen ${ }^{3}$, Ole N. Jensen ${ }^{2} \&$ Anja Groth ${ }^{1}$

During DNA replication, nucleosomes are rapidly assembled on newly synthesized DNA to restore chromatin organization. Asf1, a key histone $\mathrm{H} 3-\mathrm{H} 4$ chaperone required for this process, is phosphorylated by Tousled-like kinases (TLKs). Here, we identify TLK phosphorylation sites by mass spectrometry and dissect how phosphorylation has an impact on human Asf1 function. The divergent C-terminal tail of Asfla is phosphorylated at several sites, and this is required for timely progression through $\mathrm{S}$ phase. Consistent with this, biochemical analysis of wild-type and phospho-mimetic Asfla shows that phosphorylation enhances binding to histones and the downstream chaperones CAF-1 and HIRA. Moreover, we find that TLK phosphorylation of Asfla is induced in cells experiencing deficiency of new histones and that TLK interaction with Asfla involves its histone-binding pocket. We thus propose that TLK signalling promotes histone supply in S phase by targeting histone-free Asf1 and stimulating its ability to shuttle histones to sites of chromatin assembly.

\footnotetext{
${ }^{1}$ Biotech Research and Innovation Centre (BRIC) and Centre for Epigenetics, University of Copenhagen, Ole Maaløes Vej 5, Copenhagen DK-2200, Denmark. ${ }^{2}$ Department of Biochemistry \& Molecular Biology and Centre for Epigenetics, University of Southern Denmark, Campusvej 55, Odense DK-5230,

Denmark. ${ }^{3}$ Department of Proteomics, The Novo Nordisk Foundation Center for Protein Research, University of Copenhagen, Blegdamsvej 3B, Copenhagen DK-2200, Denmark. ${ }^{4}$ The Finsen Laboratory, Rigshospitalet, Copenhagen Biocenter, Ole Maaløes Vej 5, Copenhagen DK-2200, Denmark. †Present addresses: Novartis LCC, Leningradskiy avenue 72, 125315 Moscow, Russia (I.M.K.); Novo Nordisk Foundation Center for Biosustainablity, Technical University of Denmark, 2970 Hørsholm, Denmark (P.M.); Institute of Medical Biology, University of Tromsø, 9037 Tromsø, Norway (J.M.). Correspondence and requests for materials should be addressed to A.G. (email: anja.groth@bric.ku.dk).
} 
$\mathrm{n}$ S phase of the cell cycle, DNA must be accurately duplicated and its organization into chromatin reproduced to maintain genetic and epigenetic information. During DNA replication, chromatin organization is disrupted ahead of the replisome and restored behind, on the two daughter strands ${ }^{1-3}$. To maintain nucleosomal density, parental histone recycling must be complemented by deposition of newly synthesized histones. Duplication of chromatin thus necessitates an efficient supply of new histones, which is partly achieved by the coordination of canonical histone biosynthesis with DNA replication ${ }^{4}$.

Before their incorporation into chromatin, soluble histones are handled by histone chaperones. Asf1 is a key histone $\mathrm{H} 3-\mathrm{H} 4$ chaperone $e^{5}$ and loss of Asf1 function causes severe replication defects and loss of chromatin integrity ${ }^{6-9}$. Asf1 participates in replication-coupled nucleosome assembly by shuttling newly synthesized histone $\mathrm{H} 3.1-\mathrm{H} 4$ dimers to sites of ongoing replication. This process most likely relies on histone transfer between different chaperone complexes dedicated to histone acetylation (RbAp46-HAT1), histone nuclear import (Asf1, Importin-4) and histone storage (NASP) $)^{6,10-13}$. In the nucleus, Asf1 donates histones H3.1-H4 to CAF-1 (refs 5,14-16), which through interaction with PCNA deposits histones onto newly synthesized $\mathrm{DNA}^{17,18}$. Furthermore, Asf1 may contribute to parental histone recycling through its association with the replicative helicase MCM2-7 (refs 11,19). If replication is arrested in response to genotoxic insults, Asf1 acts as a buffer to store excess histones that cannot be incorporated into chromatin $^{6,11}$. In addition to its replication-coupled functions, Asf1 supplies replacement histone H3.3-H4 dimers to HIRA for replication-independent chromatin assembly ${ }^{20,21}$.

The mechanisms that control Asf1 activity and direct it towards distinct assembly pathways are not well understood. In humans, there are two Asfl isoforms, Asfla and Asf1b, with largely redundant roles in $S$ phase ${ }^{6,19,22}$. Both isoforms can deliver histones to CAF-1 for DNA synthesis-coupled chromatin assembly in vitro ${ }^{6,15}$, and only co-depletion of Asfla and Asf1b arrests cells in $\mathrm{S}$ phase ${ }^{6,19}$. Both isoforms bind histone $\mathrm{H} 3.1-\mathrm{H} 4$ and $\mathrm{H} 3.3-\mathrm{H} 4$ in vivo ${ }^{20}$, but Asfla appears to be the main histone donor for HIRA ${ }^{20,21,23}$. Consistent with this, Asflb is expressed only in proliferating cells ${ }^{22,24}$, while Asfla is expressed also in quiescence $^{22}$. The N-terminal domain, highly conserved between Asfla and Asf1b, forms a $\beta$-barrel structure ${ }^{25,26}$ with a hydrophobic groove accommodating the $\mathrm{H} 3-\mathrm{H} 4$ dimer and a

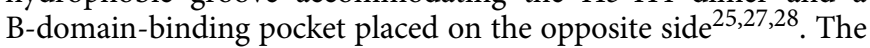
latter serves as a docking site for the downstream chaperones HIRA and CAF-1 (refs 21,26). How histone transfer occurs is poorly understood, but it may involve conformational changes in both the H3-H4 dimer and within Asf1 itself ${ }^{29,30}$. The C-terminal tail of Asf1 is unstructured and highly divergent among species and the Asfla and Asflb isoforms. The tail of yeast Asf1 contains long stretches of acidic amino acids that contribute to histone binding $9,26,31$. In higher eukaryotes, Asf1 homologues are subject to regulation by serine/threonine kinases of the Tousled-like family (TLKs) ${ }^{32}$, but the functional implications of phosphorylation have so far remained elusive. However, as the C-terminal acidic stretches are largely lost in metazoan Asf1 homologues, it has been speculated that TLK phosphorylation somehow would compensate for this ${ }^{33}$.

TLKs are $\mathrm{S}$ phase active kinases ${ }^{34}$, which are rapidly inactivated in response to genotoxic stress through phosphorylation by the checkpoint kinase Chk1 (refs 35,36). In Drosophila and C. elegans, TLK deletion causes developmental arrest due to serious defects in chromatin organization and replication ${ }^{37,38}$, while TLK depletion in human cells leads to chromosome segregation defects and aneuploidy ${ }^{39}$. Genetic analysis in flies argues that the deregulation of Asfl function is at least partly responsible for these defects ${ }^{37}$, although the underlying mechanism remains unclear. Here, we dissect how TLK phosphorylation regulates Asf1 function. Using mass spectrometry, we identify multiple TLK phosphorylation sites in the C-terminal tails of human Asfla and Asflb. Analyses of phosphorylation-deficient and phosphomimetic mutants reveal that TLK phosphorylation of Asf1 promotes $S$ phase progression and facilitates its interaction with histones and downstream chaperones in vivo. Consistent with these findings, Asf1 phosphorylation peaks in S phase and is further enhanced when the demand for new histones is high due to inhibition of histone biosynthesis. Taken together, our results suggest that TLK phosphorylates histone-free Asf1 to promote provision of histones for chromatin assembly.

\section{Results}

Identification of TLK1 phosphorylation sites in Asf1. Mass spectrometry (LC-MS/MS) was used to map phosphorylation sites in Asfla and Asf1b phosphorylated in vitro by TLK1 and in vivo in HeLa cells. We phosphorylated recombinant Asfla and Asf1b in vitro with recombinant myc-TLK1 and isolated potential phosphorylated forms of Asfl based on their slower migration in SDS-PAGE (Supplementary Figs 1a and 2a). Because of the low number of trypsin cleavage sites and high frequency of serines and threonines in the C-terminal tail of Asf1, the low specificity protease thermolysin was primarily used for digestion and precise mapping of the individual phosphorylation sites (for details see Methods). Out of twelve serine/threonines in the Asfla C-terminal tail, we identified a total of four phosphorylation sites (S166, S175, S192 and S199) (Fig. 1a and Supplementary Fig. 1). In the tail of Asf1b, we found two phosphorylation sites (S169 and S198) (Fig. 1a and Supplementary Fig. 2).

Next, we addressed whether Asf1 (a and b) phosphorylation could be detected in vivo at the sites identified in vitro. We took advantage of HeLa S3 cells stably expressing OneStrep-tagged Asfla and Asf $1 b^{19}$ to purify proteins for mass spectrometry. For Asfla, phosphorylations occurring at S166 and S175 were identified (Fig. 1b and Supplementary Fig. 3a). For Asf1b, we used cells synchronized in S phase and identified phosphorylations on S198 and T179 (Supplementary Fig. 3b). Given that pT179 was not identified after extensive phosphorylation of Asflb with myc-TLK1 in vitro, we believe that this residue is probably targeted by other kinases in vivo.

To further validate the identified phosphorylation sites, we tested Asfla and Asflb truncation and serine to alanine (S/A) mutants in a myc-TLK1 kinase assay. The level of phosphorylation decreased gradually upon shortening of the C-terminal tail of Asfla, and removal of the entire tail reduced phosphorylation by $85 \%$ compared with the wild type (Fig. 1c, compare 1-156 and wt). Single serine to alanine substitutions as well as simultaneous mutation of the sites identified in vivo (Asfla pS166, pS175) had a moderate effect (Fig. 1c, see Asfla 2A; Supplementary Fig. 4a). However, mutation of all four sites identified in vitro reduced phosphorylation to the same extent as removing the full Asfla tail (Fig. 1c, compare Asf1a 4A and 1-156). Similarly, substituting both serines identified in vitro (Asf1b pS169, pS198) or truncation of the C-terminal tail (Asf1b 1-157) eliminated phosphorylation of Asf1b (Fig. 1c, compare Asf1b 2A, 1-157 and wt). Taken together, our mapping analysis argues that TLKs can target four sites in Asfla and two sites in Asflb, all located within the divergent C-terminal tails.

To study Asfla phosphorylation in vivo, we generated antibodies against all sites in Asfla and Asf1b that had reasonable immunogenic properties (Asf1a pS166, pS175, pS192; Asf1b pS169). Of these, only the antibody against Asfla pS166 showed high specificity. It recognized wild-type Asfla phosphorylated by 
myc-TLK1 in vitro but not the S166A mutant or the unphosphorylated wild-type protein (Fig. 1d). In total cell lysates, the pS166 antibody detected two bands, which correspond to slow migrating forms of Asfla (Fig. 1e). It should be noted that the resolution of these phospho-forms depends on the SDS-PAGE conditions and are not always easily discernible. Importantly, the pS166 signal was enhanced upon overexpression of wild-type myc-TLK1, while kinase-inactive (ki) myc-TLK1 markedly reduced both phospho-forms (Fig. 1f). Thus, the pS166 antibody specifically detects TLK phosphorylation of Asfla at S166, and the two bands probably represent differentially phosphorylated forms of Asfla that have pS166 in common.

Asf1a pS166 is nuclear and peaks during DNA synthesis. TLKs are hyperactive in $S$ phase ${ }^{34}$ and rapidly inactivated upon DNA damage or replication stress through direct phosphorylation by Chk1 (refs 35,36). We thus used the pS166 antibody to monitor TLK1 phosphorylation of Asfla during the cell cycle and after treatment with replication inhibitors. Analysis of synchronized U-2-OS cells showed that Asfla S166 phosphorylation peaks in mid S phase and decreases in late S and G2 phase (Fig. 2a). Consistent with this, immunofluorescence analysis revealed nuclear Asfla pS166 staining in most replicating cells marked by EdU (Fig. 2b). However, a fraction of pS166-positive cells did not incorporate EdU. While some of these might represent cells at the G1/S transition, others were in mitosis when TLK2 is known to have moderate activity ${ }^{32,34}$. No staining was seen in Asfladepleted cells, verifying the specificity of the antibody (Fig. 2b). This confirms that the slow migrating phospho-forms of Asfla previously observed in $S$ phase ${ }^{32}$ reflect TLK-mediated phosphorylation. Inhibition of DNA replication by hydroxyurea (HU) reduced Asf1 S166 phosphorylation by $60-85 \%$ within $1 \mathrm{~h}$, as shown by western blot, immunoprecipitation and immunostaining (Fig. 2a,c and d). Concomitant treatment with the Chk1 inhibitor UCN-01 partially rescued Asf1 pS166 (Fig. 2c). Yet, the effect of checkpoint abrogation was modest, suggesting that checkpoint-independent events may contribute to the loss of Asfl phosphorylation upon a replication block (see discussion). Nonetheless, Chk1 inhibition augmented Asfla pS166 in unperturbed S phase cells (Fig. 2c,d), indicating that Asf1 phosphorylation, similar to TLK activity ${ }^{35}$, is controlled by basal Chk1 activity in $S$ phase.

TLK1 phosphorylation of Asfla facilitates S-phase progression. To explore the role of Asf1 phosphorylation in S phase, we established U-2-OS Flp-In cells conditionally expressing FLAGHA-Asfla (e-Asfla) wild-type (wt), a phosphorylation deficient (4A) mutant or a phospho-mimetic mutant (4D), all rendered insensitive to siRNAs targeting endogenous Asfla. We chose to mutate all four sites identified with high confidence in vitro, since this reduced TLK phosphorylation by $85 \%$ similar to removing the whole C-terminal tail (Fig. 1c). A recent study found that TLK2 can phosphorylate Asfla at S192 in vitro and proposed that phosphorylation of this single site stabilizes Asfla by preventing proteasomal degradation ${ }^{40}$. We therefore tested the stability of our Asfla phospho-mimetic (4D) and phosphorylation deficient (4A) mutants in cycloheximide (CHX)-treated cells. We did not observe significant differences in stability (Supplementary Fig. 4b). However, we noted that CHX treatment strongly stimulated Asf1 phosphorylation (Supplementary Figs 4b, 6b), which due to the mobility shift might influence stability measurements of the wild-type protein.

Overexpression of either Asfla wild-type or the phosphomutants did not significantly perturb the cell cycle (Supplementary Fig. 4c). We thus asked whether the mutants could substitute for endogenous Asfla and rescue cells arrested in $S$ phase due to co-depletion of Asfla and Asf1b (Fig. 3). We chose to deplete both isoforms, as single depletions do not efficiently block $S$ phase progression ${ }^{22}$. Western blotting confirmed the efficient depletion of endogenous Asf1 ( $a$ and $b$ ) and equal expression of e-Asfla proteins (Fig. 3a). In the absence of Asf1 ( $\mathrm{a}$ and $\mathrm{b}$ ), approximately $50 \%$ of the cells were in $\mathrm{S}$ phase, as compared with $25 \%$ of the control cells (Fig. 3b,c). Short-term expression of wild-type e-Asfla reduced the number of the $\mathrm{S}$ phase cells to $35 \%$, and the phospho-mimetic mutant (4D) had similar or better rescue efficiency (Fig. 3b). In contrast, the e-Asfla 4A mutant showed significantly lower rescue activity (Fig. 3b,c, Supplementary Fig. 4d). Similarly, complementation of synchronized cells illustrated that phospho-mimetic Asfla accelerated S-phase progression most efficiently, while the phospho-deficient mutant was inferior to wild-type Asfla (Fig. 3d,e, Supplementary Fig. 4e,f). This indicates that TLK phosphorylation promotes Asf1 function in $S$ phase.

Phosphorylation promotes complex formation with histones. Next we explored whether phosphorylation influences the properties of Asfl as a histone chaperone by comparing complex composition of wild-type Asfla against the phosphorylationdeficient (4A) and phospho-mimetic (4D) mutants. We used our conditional cell lines to purify complexes from cells synchronized in mid S phase. Comparison of cytosolic complexes, representing highly soluble cytoplasmic and nuclear Asf1 (ref. 11), showed that the phospho-mimetic Asfla mutant binds more histone H3, new histone $\mathrm{H} 4$ acetylated at lysine 12, Importin-4, NASP and RbAp46/48 than wild-type Asfla (Fig. 4a). Importin-4, NASP and $\mathrm{RbAp} 46 / 48$ form a complex with Asf1 through mutual binding to histone $\mathrm{H} 3-\mathrm{H} 4$ (ref. 11), and their abundances are thus expected to mirror histone binding as we observe. Previous work has shown that Asfl distributes into a histone-bound multichaperone complex and a histone-free form in cytosolic extracts from $S$ phase cells ${ }^{6}$. Analysis of the phospho-mutants by gel-filtration demonstrated a clear shift of the Asfla phospho-mimetic mutant (4D) towards the histone-bound complex relative to the wild-type and $4 \mathrm{~A}$ proteins (Fig. 4b, Supplementary Fig. 5a). Consistent with this, S166 phosphorylation on endogenous Asfla could also be detected in the histone-bound complex (Supplementary Fig. 5b). Moreover, TLK1 phosphorylation of GST-Asfla enhanced histone H3-H4 binding in cell extracts (Supplementary Fig. 5c). These data imply that phosphorylated Asf1 might bind soluble cellular histones more efficiently. Kinetic rate constants between recombinant Asfla and immobilized histone $\mathrm{H} 3-\mathrm{H} 4$ in vitro were therefore determined by surface plasmon resonance (Supplementary Fig. 6). The derived rate constants translates into a very high affinity $\left(K_{\mathrm{d}}=46 \pm 24\right.$ pM, Supplementary Fig. 6) for the interaction between wild-type Asfla and histones, which align very well with previous data recorded at equilibrium ${ }^{30}$. However, using these experimental conditions we could not detect significant differences between wild-type Asfla and the phospho-mimetic mutant. It is, nevertheless, possible that the electrostatic immobilization of $\mathrm{H} 3-\mathrm{H} 4$ on the sensor chip sterically prevents an interaction with the acid tail of phospo-mimetic Asf1, thus providing a suboptimal surrogate for cellular histones. We therefore devised a competition assay to compare the binding of recombinant wild-type and phospho-mimetic proteins to histones in a cell extract. Recombinant Asfla wild-type and phospho-mimetic mutant (4E) were added together to an extract of HeLa S3 cells expressing H3.1-HA-FLAG (e-H3.1). Upon isolation of e-H3.1 complexes, we found a significant enrichment of the phosphomimetic mutant relative to recombinant wild-type Asfla (Fig. 4c). 


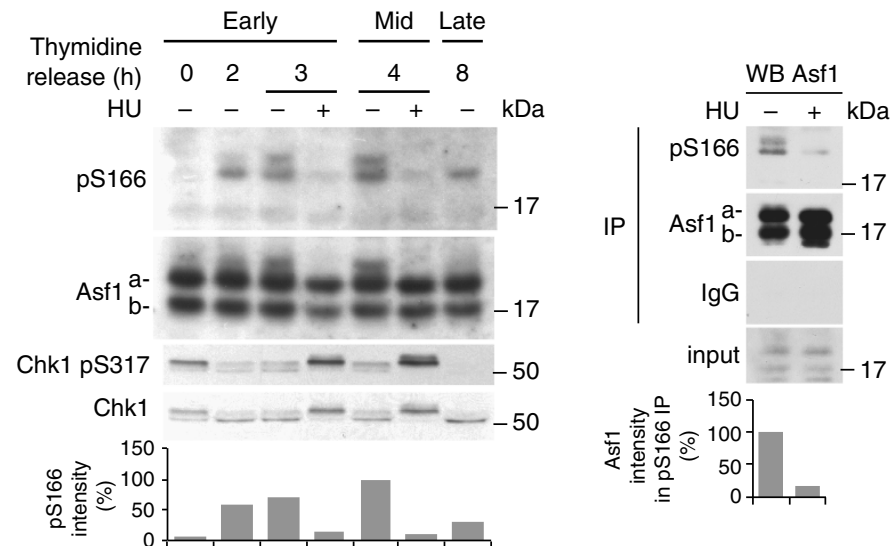

b
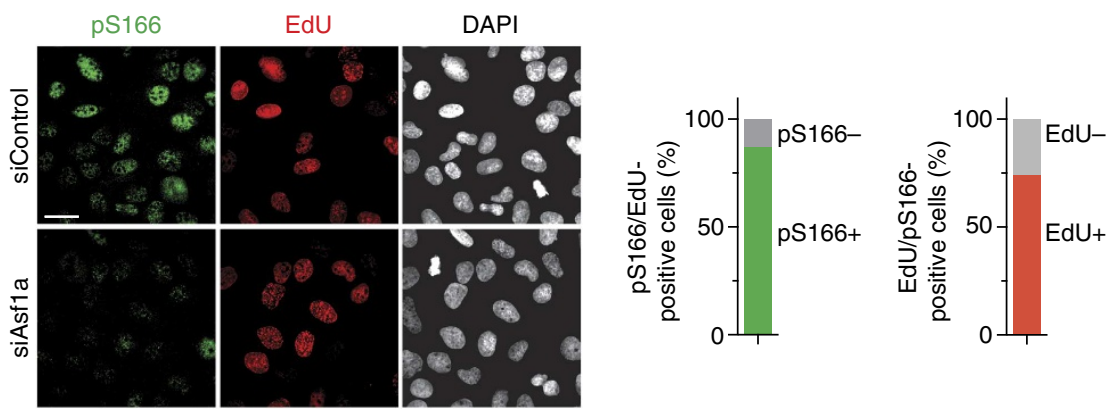

C

d

HU

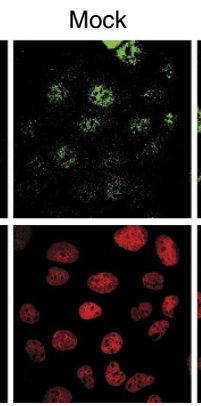

UCN-01
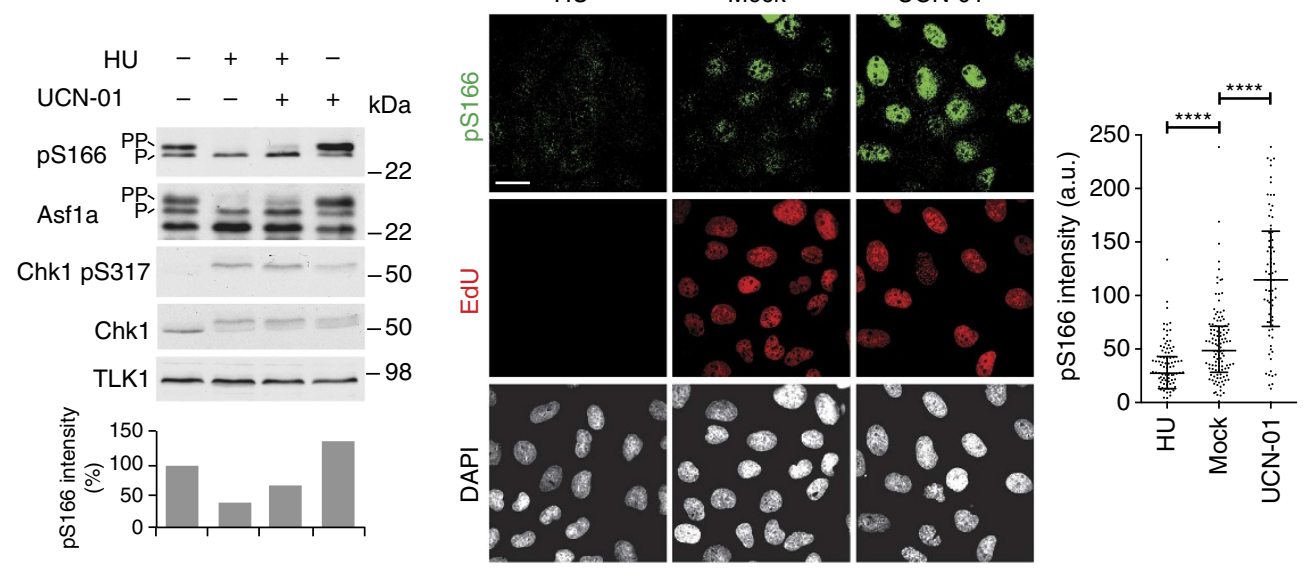

Figure 2 | Asf1a pS166 is nuclear and peaks during ongoing DNA synthesis. (a) U-2-OS cells were synchronized by a thymidine block at the G1/S boundary and released into S-phase (early S, mid S, late S/G2). Parallel cultures were treated for one hour with HU, as indicated. (Left) pS166 western blot. (Right) Immunoprecipitation with pS166 and Asf1 ( $a$ and b) antibodies followed by western blot for Asf1. Graphs show relative pS166 or Asf1 signal intensities. One representative experiment out of two biological replicas is shown. (b) Immunofluorescence analysis of Asf1a pS166 in siRNA treated U-2-OS cells. Replicating cells were labelled with EdU. Graphs depict the percentage of pS166-positive/negative cells in the EdU-positive population and vice versa. More than 120 cells per sample were analysed. Scale bar, $32 \mu \mathrm{m}$. (c) U-2-OS cells synchronized in early S phase as in (a) were treated with $\mathrm{HU}$ and/or UCN-01 for $1 \mathrm{~h}$. Relative pS166 signal intensities are depicted in the graph. One representative experiment out of two biological replicas is shown. (d) Immunofluorescence analysis of Asfla pS166 in cells treated as in (c). Replicating cells were labelled with EdU. Scale bar, $32 \mu \mathrm{m}$. Graph shows pS166 intensities, where median and interquartile range are indicated, $n>70,{ }^{\star \star \star \star} P<0.0001$ (unpaired $t$-test). P and PP denote different Asf1a phosphorylation states.

Importantly, this was paralleled by a larger depletion of the phospho-mimetic mutant from the unbound fraction. Asfla containing a mutated histone-binding pocket $(\mathrm{V} 94 \mathrm{R})^{25}$ did not bind histones in the extract.

To address whether phosphorylation also affected the interaction with Asf1 downstream chaperones, CAF-1 and HIRA, we analysed complexes from nuclear extracts made by high-salt extraction of chromatin ${ }^{19}$. The nuclear e-Asfla $4 \mathrm{D}$ complexes showed increased association to both non-nucleosomal histone H3-H4 and the two downstream chaperones CAF-1 and HIRA (Fig. 5a). To corroborate these findings, we used SILAC-based mass spectrometry to quantitatively compare the composition of wild-type and phospho-mimetic Asfla complexes isolated from whole-cell extracts. This approach confirmed our western blot 


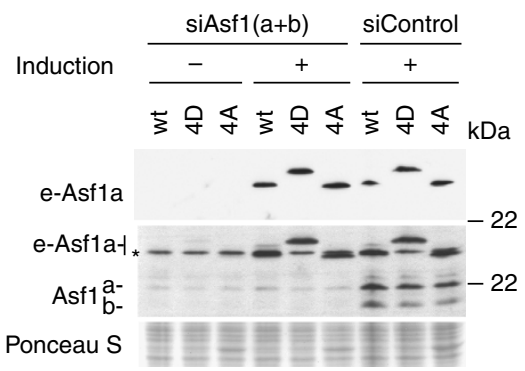

b

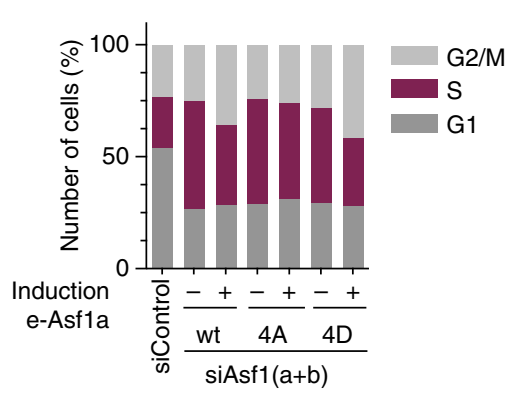

c

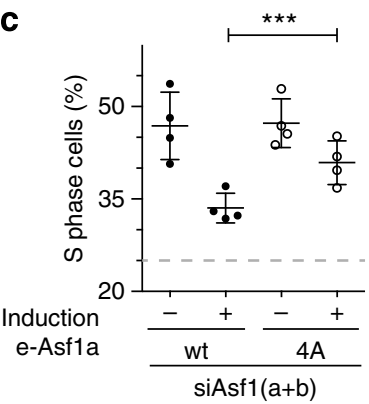

d

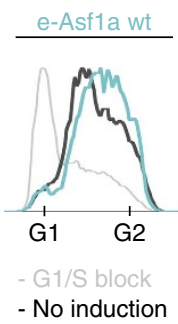

e

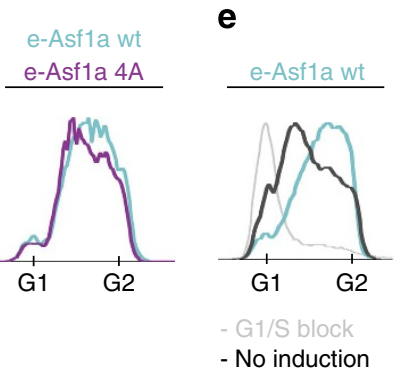

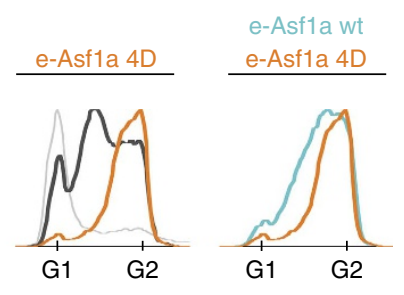

G2

Figure 3 | TLK1 phosphorylation of Asf1a facilitates S phase progression. (a) (Left) Experimental design for complementation analysis. (Right) Western blot showing induction of siRNA-resistant forms of e-Asfla wt/4D/4A after depletion of endogenous Asf1 a and b (siAsf1( $a+b)$ ). *, unspecific band.

(b) Quantification of cell cycle distribution. Cell cycle profiles are shown in Supplementary Fig. 4b. (c) Quantification of $S$ phase cells in 4 independent experiments comparing the complementation efficiency for e-Asfla wt vs. $4 \mathrm{~A}$. The mean with s.d is shown; ${ }^{\star \star \star} P=0.0008$ (unpaired $\mathrm{t}$ test). The dashed line shows average percentage of $\mathrm{S}$ phase cells in siControl cells. (d,e) Complementation analysis in synchronized cells. Complementation of endogenous Asf1 ( $a$ and $b$ ) was assessed $9 \mathrm{~h}$ after release from a G1/S arrest. Asf1a proteins were expressed at similar levels and endogenous Asf1 ( $a$ and b) were efficiently depleted (Supplementary Fig. 4e,f). Light grey lines, control cells in G1/S; dark grey lines, Asf1 (a and b)-depleted cells without induction; colored lines, Asf1 ( $a$ and b) depleted cells complemented with e-Asfla wt (blue), 4A (purple) and 4D (orange).

data, showing up to twofold more histone H3-H4, Importin-4, NASP, RbAb46 and HIRA in e-Asfla $4 \mathrm{D}$ complexes (Fig. 5b). We did not detect CAF-1 in the SILAC analysis, likely reflecting that this interaction is weak compared with HIRA binding 21 . However, analysis of Asflb complexes showed that, although histone $\mathrm{H} 3-\mathrm{H} 4$ binding was not markedly changed, the phospho-mimetic mutations clearly favoured association with CAF-1 (Fig. 5c).

The unstructured C-terminal tails of Asfla and Asflb are approximately 50 amino acids long and extrude from the globular domain between the histone-binding pocket and the B-domain binding surface ${ }^{25-28}$. To address how the tail might influence interactions at these sites, we performed a series of pull-down experiments using truncated versions of GST-Asfla. Tail-less Asfla retrieved histones, histone-dependent interactors (NASP, RbAp46/48) as well as CAF-1 and HIRA more efficiently than the full-length protein from HeLa S3 cell extracts (Fig. 5d). Furthermore, isolation of complexes from transiently transfected cells revealed that removal of only 30 amino acids from the Asfla tail was sufficient to improve complex formation with histones and NASP (Fig. 5e).
Histone deficiency induces TLK phosphorylation of Asf1. In our GST pull-down experiments, we noted that tailless Asfla bound efficiently to TLK1 (Supplementary Fig. 7a). Furthermore, we found that an intact histone-binding pocket, but not the B-domain binding site, is required to pull-down TLK1 from cell extracts (Supplementary Fig. 7a). This suggests that TLK1 either interacts directly with the histone-binding pocket in Asf1 or binds via histone $\mathrm{H} 3-\mathrm{H} 4$. Consistent with the first scenario, in vitro binding reactions with recombinant proteins showed that mycTLK1 failed to bind Asf1a V94R (Fig. 6a). This implies that TLK1 docks onto Asf1 via the histone-binding pocket and thus predicts that histone-free Asf1 would be the preferred substrate. In this case, Asf1 phosphorylation should increase when the level of soluble histones is low. We therefore investigated Asfla phosphorylation status in cells experiencing a deficiency of new soluble histones. Since histones produced in $S$ phase are rapidly incorporated into chromatin, short-term inhibition of protein synthesis by CHX is routinely used to reduce the pool of new soluble histones ${ }^{41,42}$. Asfla phosphorylation, detected by the pS166 antibody or mobility shift, increased in S phase cells after short-term CHX treatment (Fig. 6b). Moreover, depletion of two 
a

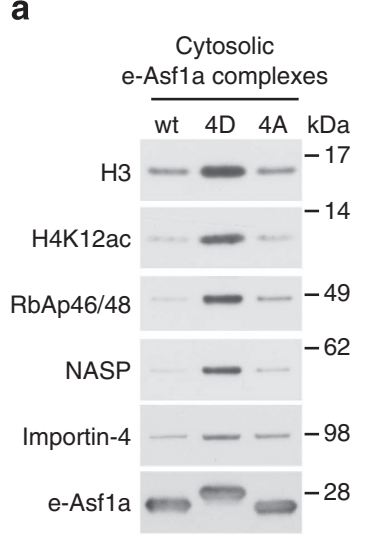

C

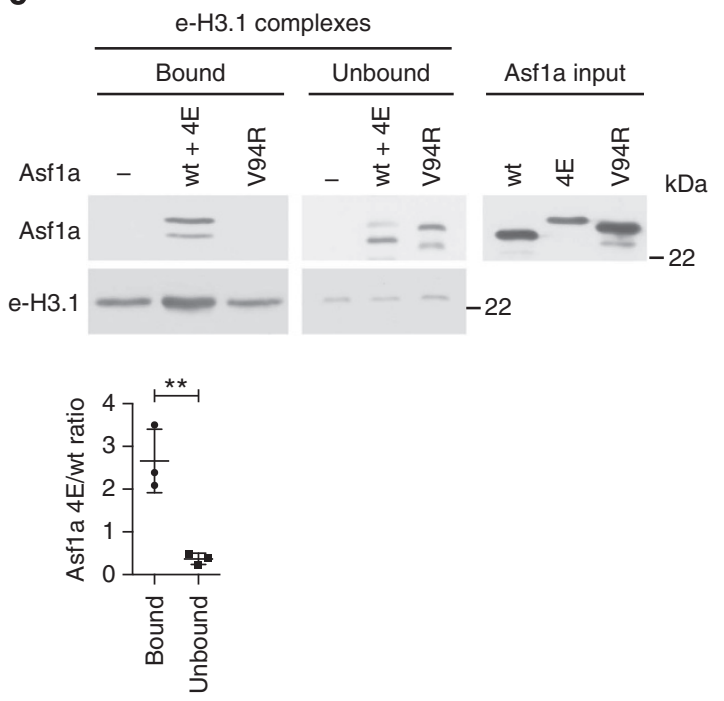

b
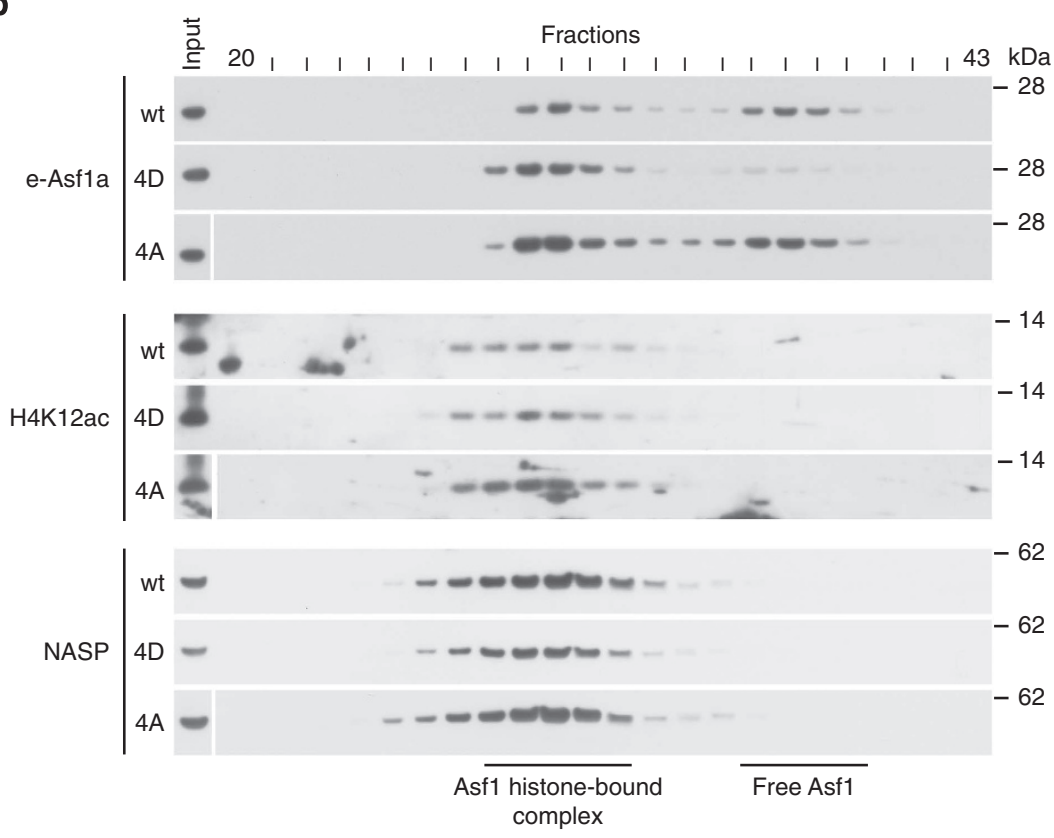

Figure 4 | Phosphorylation enhances Asf1 binding to soluble cellular histones. (a) Western blot analysis of e-Asfla complexes isolated from conditional cell lines expressing e-Asfla wt/4D/4A. Cytosolic complexes were obtained from cells released into mid-S phase from a thymidine block. (b) Gel-filtration analysis of cytosolic extracts from S phase cells conditionally expressing e-Asf1a wt/4D/4A. Similar induction of e-Asfla wt/4D/4A was verified (Supplementary Fig. 5a). Two distinct Asf1 complexes are indicated. (c) In vitro competition assay. Whole-cell extracts of HeLa S3 cells expressing H3.1-HA-FLAG ${ }^{20}$ were pre-incubated with mixture of recombinant Asfla wt and phospho-mimetic (4E) mutant or with Asf1a V94R. e-H3.1 complexes were purified and binding of recombinant Asf1a assessed taking advantage of the difference in mobility between wt and 4E. The ratio of Asfla 4E relative to wt in the bound and unbound fractions was quantified in three independent experiments as depicted in the graph. The mean with s.d is shown, ${ }^{\star \star} P=0.0063$ (unpaired $t$-test).

key factors required for canonical histone biosynthesis, FLASH and SLBP ${ }^{43,44}$, also enhanced Asfla phosphorylation in S phase (Fig. 6b, Supplementary Fig. 7b), substantiating the link between lack of soluble histones and TLK phosphorylation of Asf1.

Asf1 is mainly found in the nucleus where it distributes into highly mobile (soluble) and chromatin-bound complexes $6,11,19$. We noted that hyper-phosphorylated Asfla was enriched relative to the unphosphorylated form in chromatin when compared with the soluble fraction (Fig. 6c). Analysis of TLK1 revealed that a fraction of the kinase also remains in chromatin after extraction with Triton (Fig. 6c). Intriguingly, the level of chromatin-bound
TLK1 increased when histone biosynthesis was impaired by short-term CHX treatment or FLASH/SLBP depletion (Fig. 6d,e). In contrast, TLK1 was released from chromatin in response to replication stress (Fig. 6d). Thus, TLK1 binding to chromatin correlates with Asf1 phosphorylation status (Fig. 6b,d and e). Asfla pS166 could be detected in chromatin by western blot (Fig. 6d) but not by immunofluorescence. However, taking advantage of a GFP-TLK1 conditional cell line, we could address the localization of the kinase. As expected ${ }^{34}$, GFP-TLK1 was mainly nuclear. Yet, pre-extraction revealed two populations of cells; one with a diffuse nuclear signal and another showing 
a

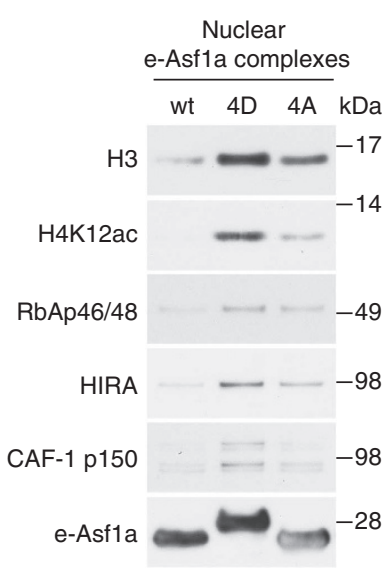

C

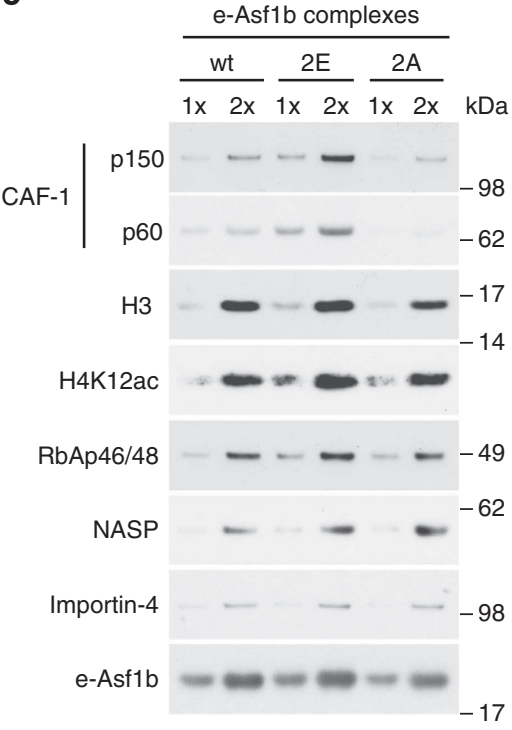

b

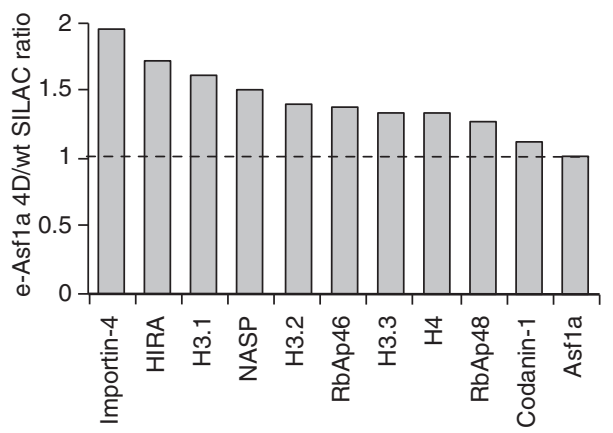

d

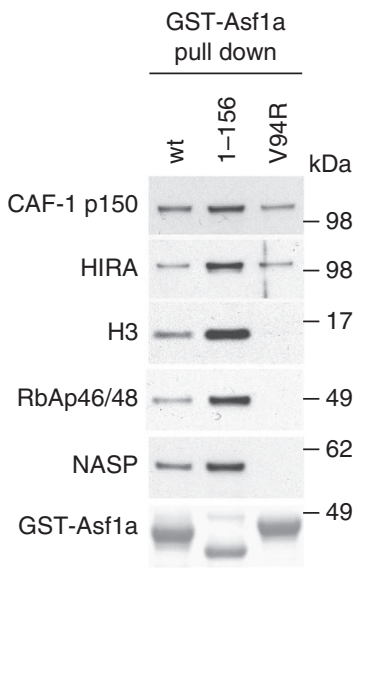

e

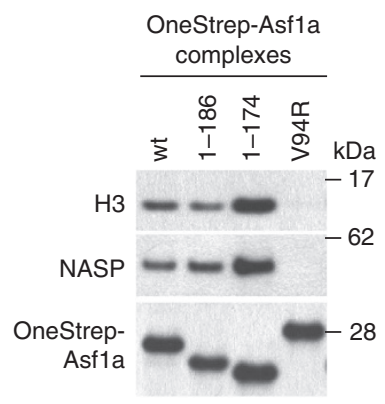

Figure 5 | Phosphorylation promotes Asf1 complex formation with downstream chaperones. (a) Western blot analysis of e-Asfla complexes isolated from conditional cell lines expressing e-Asf1a wt/4D/4A. Nuclear extracts were prepared from cells synchronized as in Fig. 4a.

(b) SILAC-based proteomic analysis of e-Asfla 4D and wt complexes. Complexes were isolated from whole extracts of conditional cells grown in heavy (e-Asfla 4D) and light (e-Asfla wt) amino acids, mixed in a 1:1 ratio and analysed by mass spectrometry. The relative enrichment in e-Asf1a $4 \mathrm{D}$ versus wt complexes of known binding partners is indicated with raw ratios. A dashed line indicates no enrichment. The result of a representative experiment out of three biological replicates is shown. (c) Complexes containing Flag-HA-Asf1b (e-Asf1b) wt, phospho-mimetic (2E: S169E and S198E) and phosphorylation-deficient (2A: S169A and S198A) mutants were affinity purified from whole cell extracts of transiently transfected U-2-OS cells. (d) Tail-deletion augments Asf1 binding to soluble histones. Pull-down using GST-Asfla full length (wt), truncated (1-156) or histone-binding (V94R) mutant as bait in U-2-OS cell extracts. (e) Complexes containing OneStrep-Asfla wt, truncations $(1-174,1-186)$ or V94R mutant purified from whole-cell extracts of transiently transfected U-2-OS cells.

distinct nuclear foci (Supplementary Fig. 7c). Co-staining with PCNA to mark replication sites revealed that non-S phase cells generally showed diffuse distribution of GFP-TLK1 in chromatin. In contrast, GFP-TLK1 localized in foci overlapping with PCNA replication patterns in the majority of $S$ phase cells (Fig. 6f). 
a

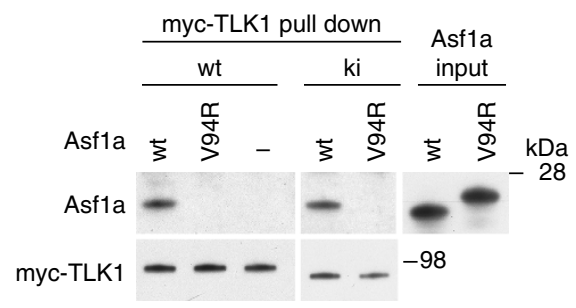

b

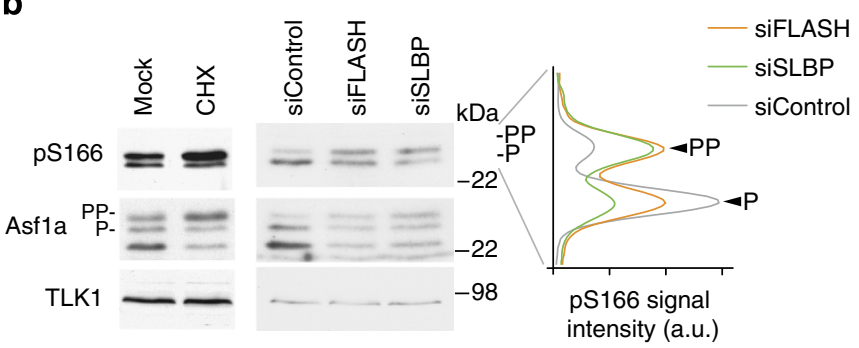

c

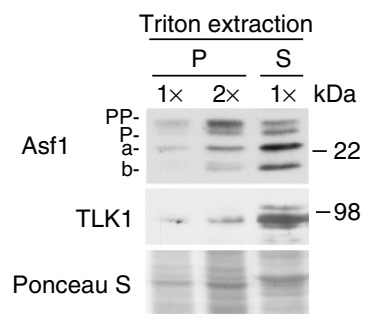

d

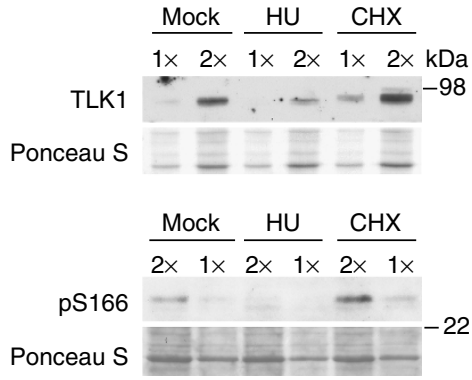

e

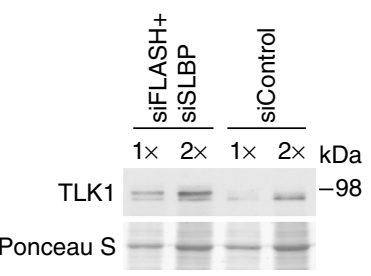

$f$
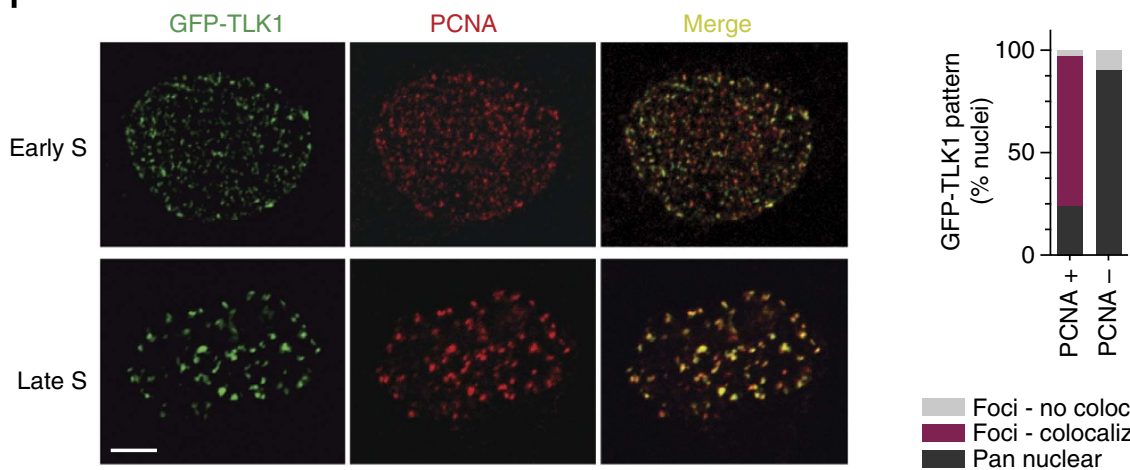

Foci - no colocalization
Foci - colocalization

Faci - coloca

Figure 6 | TLK1 localizes to replication sites and phosphorylates histone-free Asf1. (a) In vitro binding assay. Recombinant Asfla wild-type (wt) or histone-binding mutant (V94R) was incubated with myc-TLK1 wild-type (wt) or kinase-inactive (ki) isolated from insect cells. (b) Western blot of Asf1a phosphorylation in S phase cells treated with $\mathrm{CHX}$ for one hour or siRNAs against FLASH and SLBP (siFLASH, siSLBP). Graph was generated as in Fig. 1f. One representative experiment out of two biological replicas is shown. (c) Distribution of TLK1 and Asf1 between the Triton soluble (S) fraction and chromatin pellet (P) in S phase cells. P and PP denote different Asf1a phosphorylation states. (d,e) Analysis of TLK1 and Asf1a pS166 in the chromatin pellet in cells treated with $\mathrm{HU}$ and CHX for one hour (d) or siRNAs against FLASH and SLBP (siFLASH + siSLBP) (e). (f) GFP-TLK1 localization analysed in conditional U-2-OS cells by co-staining with PCNA after pre-extraction of soluble proteins with Triton. Representative images of early and late $S$ phase cells are shown. Scale bar, $5 \mu \mathrm{m}$. The graph shows quantification of GFP-TLK1 localization patterns in PCNA-positive $(+)$ and PCNA-negative $(-)$ cells. One representative experiment out of two biological replicas is shown $(n>100)$.

\section{Discussion}

Here we identify multiple TLK phosphorylation sites in human Asfla and Asflb (Fig. 7a) and provide evidence that phosphorylation stimulates the association of Asfla with histones and downstream chaperones in vivo. We propose that TLK signalling promotes histone provision in $\mathrm{S}$ phase by targeting histone-free Asf1 and stimulating its ability to shuttle histones to sites of chromatin assembly (Fig. 7b). The key pieces of evidence in support of this model are (i) Asfla phosphorylation is required for timely progression through $S$ phase, (ii) phospho-mimetic Asfla shows superior ability to form cellular complexes with histones and downstream chaperones, (iii) TLK1 binds to Asfla via the same pocket as histone $\mathrm{H} 3-\mathrm{H} 4$ and (iv) TLK1 recruitment to chromatin and Asfla phosphorylation is induced in cells experiencing deficiency of new histones.

Most of the TLK1 phosphorylation sites we identify in human Asfla and Asf1b are conserved in vertebrates (Fig. 7a). A few of these sites have been found in large phospho-proteomic analyses $(\text { S192, S166) })^{45,46}$, and Asf1a S192 and Asf1b S198 were recently reported to be phosphorylated in vitro by TLK2 (ref. 40). It was proposed that S192 phosphorylation stabilizes Asfla (but not Asflb) by preventing proteasomal degradation ${ }^{40}$. We did not see changes in protein stability upon mutation of multiple Asfla phosphorylation sites into either aspartic acids or alanines. However, we noticed that cycloheximide treatment stimulates phosphorylation of wild-type Asfla considerably, which given the mobility shift of the phospho-forms may influence stability measurements. We also note that, while phosphorylation peaks in $S$ phase, Asfla protein levels remain relatively constant during the cell cycle ${ }^{22,32}$. Our data propose that TLK phosphorylation augments the ability of Asf1 to take up histones and engage with downstream chaperones when histone demand is high during replication. Since the acidic tail of S. cerevisiae Asf1 (scAsf1) contributes to histone binding and association with the heterotrimeric CAF-1 complex in vitro ${ }^{26,29,31}$, our results support the idea that TLK phosphorylation compensate for 
a

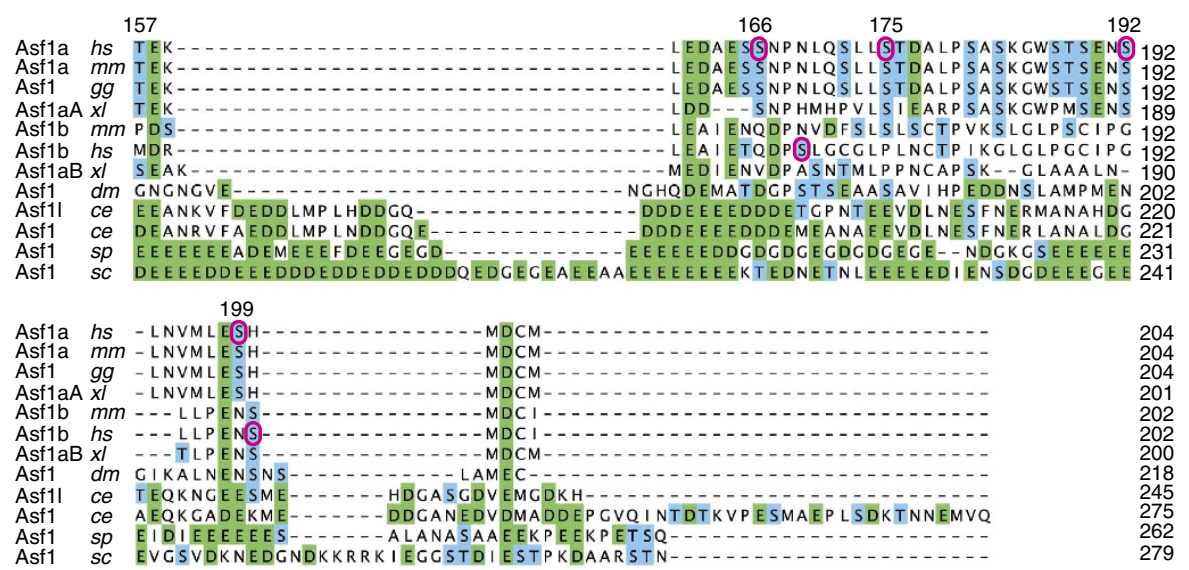

b

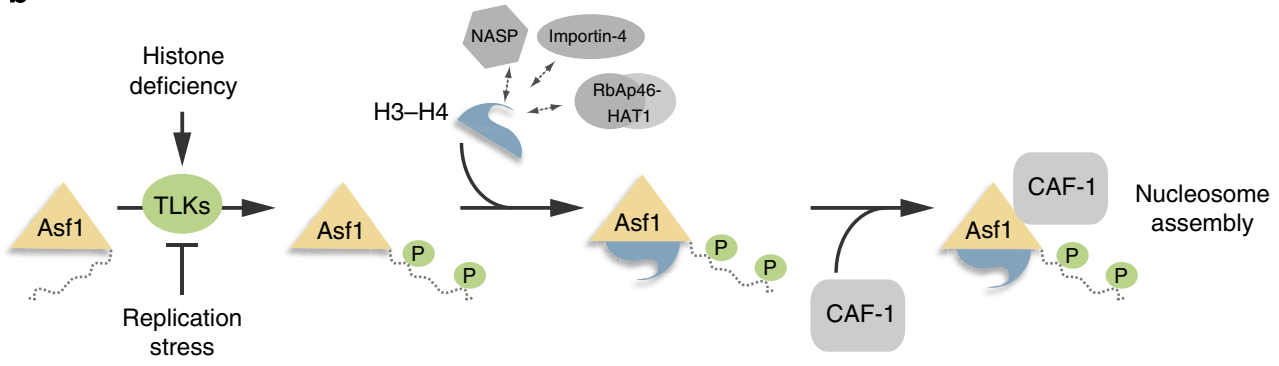

Figure 7 | Phosphorylation site conservation and model. (a) Sequence alignment of the Asf1 C-terminal tails performed by the ClustalW2 algorithm. Homo sapiens, hs; Mus musculus, mm; Gallus gallus, gg; Xenopus laevis, xl; Drosophila melanogaster, dm; Caenorhabditis elegans, ce; Saccharomyces cerevisiae, sc; Schizosaccharomyces pombe, sp. Acidic residues are indicated in green and Ser/Thr residues are in blue. Purple circles denote TLK1 phosphorylation sites in human Asf1a and Asf1b identified in this study. (b) Hypothetical model for how TLK phosphorylation fine-tunes Asf1-dependent histone provision during genome duplication. TLKs bind histone-free Asf1 and phosphorylate the tail at multiple sites. This in turn facilitates complex formation with histones and the downstream chaperones HIRA and CAF-1. TLK signalling responds to histone demand; Asfla phosphorylation increases when cells lack new soluble histones and decreases if DNA synthesis is blocked and histones demand drops.

the lack of C-terminal acidic stretches in metazoan Asf1 homologues ${ }^{33}$. In yeast the tail confers an advantage to $s c$ Asf1 in silencing ${ }^{47}$, but tailless $s c$ Asfl 1 rescues the cell cycle defects and sensitivity to genotoxic stress of asf1 mutants ${ }^{26}$. Similarly, our data imply that TLK phosphorylation is not critical for Asf1 function, but makes it a more powerful histone chaperone better able to meet the demand for histones in S phase. Phosphorylation of the four C-terminal sites in Asfla only moderately affects the charge of the full-length protein (Supplementary Fig. 5d), while the acidic tail of yeast Asfl influences the pI substantially. However, considering the Asfla tail alone, phosphorylation reduces the charge substantially and even more so than the phospho-mimetic mutations (Supplementary Fig. 5d). Our biochemical data show that multiple tail phosphorylations in Asfla promote complex formation with histone $\mathrm{H} 3-\mathrm{H} 4$, histonedependent partners like NASP and Importin-4, and the downstream chaperones HIRA and CAF-1. Similar to phosphomimetic mutations, removal of the unmodified Asfla tail augments histone binding in cells and cell extracts. Thus, the simplest model is that phosphorylation alleviates a negative effect of the unstructured tail. In addition, it is plausible that the phosphorylated tail might aid histone binding directly. We could not measure significant differences in binding rate constants for the interaction between histone $\mathrm{H} 3-\mathrm{H} 4$ and Asfla wild-type, phospho-mimetic and tailless proteins. Nevertheless, wild-type Asf1 shows very high affinity towards $\mathrm{H} 3-\mathrm{H} 4$, which makes it challenging to identify a potential additional contribution of the acidic tail. Moreover, we cannot exclude that electrostatic immobilization of $\mathrm{H} 3-\mathrm{H} 4$ obstructs an interaction with the acid tail of phospo-mimetic Asf1. Further biophysical and structural studies are thus required to resolve exactly how phosphorylation enables Asf1 to bind histones more efficiently in a cellular context.

Asfla and Asflb are found in complex with both histones H3.1-H4 and H3.3-H4 (ref. 20). Both histone variants can be deposited in S phase; the predominant replication-coupled pathway involves CAF-1 (refs 48,49), while HIRA contributes with gap-filling and histone replacement independent of replication ${ }^{48,50}$. Asfla and Asflb can both interact with CAF-1 (refs 15,20) and provide histones for replication-coupled nucleosome assembly ${ }^{6}$. In contrast, in vitro and in vivo data suggest that Asfla would be the main histone donor for HIRA $^{20,21,23}$. Phosphorylation does not appear to guide the choice of specific assembly pathway, as it enhances association of Asfla with CAF-1 and HIRA as well as Asflb binding to CAF-1. It remains unclear how histones are transferred to HIRA and CAF-1. Histone binding may itself promote Asf1 interaction with its downstream chaperones, as histone $\mathrm{H} 3-\mathrm{H} 4$ binding to scAsfl changes the $\mathrm{B}$-domain binding pocket to facilitate interaction with CAF-1 p60 (Cac2) in vitro ${ }^{29}$. This would be consistent with the enhanced association of phospho-mimetic Asfla with CAF-1 and HIRA in human cells, and explain why neither in vitro phosphorylated Asfla nor the phospho-mimetic mutant show enhanced binding to recombinant CAF-1 p60 in vitro (ref. 15, our unpublished results). Our SILAC analysis shows that phosphorylation does not increase the binding of Asfla to Codanin-1, which also binds Asf1 via a B-domain and negatively regulates Asf1-H3-H4 nuclear import ${ }^{51}$. This suggests that phosphorylation is not beneficial for all B-domain containing binding partners but favours those that promote histone deposition. In vitro, scCAF-1 and scAsf1 do not reside together 
in a stable complex when both chaperones are bound to histones ${ }^{29}$. Consistent with this, Asf1 binding to CAF-1 in human cells is weak and lost upon high-salt washes (Jasencakova and Groth, unpublished). The simplest explanation for our observation is that phosphorylation favours formation of a complex where histone $\mathrm{H} 3-\mathrm{H} 4$ has not yet been transferred from Asf1 to CAF-1. The work of Liu and colleagues ${ }^{29}$ suggests that Asf1 would be released once histone transfer takes place. Future studies should address the possibility that de-phosphorylation might trigger this event. Indeed, if phosphorylation promotes histone uptake and formation of a (H3-H4)-Asf1-CAF-1 complex, de-phosphorylation might in turn favour release of histones from Asf1.

The assembly of newly synthesized DNA into nucleosomes is central for maintaining chromatin organization and genome integrity in dividing cells. The tight coupling of histone biosynthesis to DNA replication is well established ${ }^{4}$. Our data argue that TLK signalling provides an additional means to regulate histone provision. We find that TLK1 binding to Asf1 requires an intact histone-binding pocket, and, consistent with a mutually exclusive interaction, TLK1 can compete with histones for Asf1 binding in vitro ${ }^{52}$. Phosphorylation of Asf1 and/or TLK itself has been suggested to trigger their dissociation in vitro ${ }^{37}$. However, in cells the interaction appears to be dynamic and only kinase inactive TLKs form a stable complex with Asf1 (ref. 32). Recently, the S. cerevisiae checkpoint kinase Rad53 was shown to contact the histone-binding pocket in scAsf1 (ref. 53). This analogy between TLKs and Rad53 is intriguing and supports the idea that, although TLKs do not share homology with Rad53, these kinases may have evolved as an extra regulatory layer replacing direct control of Asf1 function by checkpoint kinases. TLK activity is high in S phase when histone demand peaks, and is rapidly reduced by checkpoint signalling if replication is inhibited $^{35}$ and histone demand drops ${ }^{6}$. TLK activity would thus respond to ongoing DNA synthesis and histone demand in two ways; (i) via checkpoint signalling and (ii) by preferentially targeting histone-free Asf1. Consistent with this, Asf1a pS166 phosphorylation is enhanced in cells lacking new soluble histones for chromatin assembly and reduced in response to replication stress. Control of Asfl phosphorylation by both checkpoints and histone pools probably explains why Chk1 inhibition alone is not sufficient to rescue Asfl phosphorylation in $\mathrm{HU}$ treated cells. TLK binding to chromatin correlates with Asfla pS166 in response to stresses such as depletion of nucleotide and soluble histone pools. Moreover, TLK1 is present at sites of ongoing replication. We thus speculate that Asf1 phosphorylation could, at least in part, take place in chromatin at replication sites. The simplest scenario would be that Asf1, once it has delivered its histone cargo to CAF-1, becomes a target for TLK phosphorylation, which in turn fuels recruitment of additional histones to active replication forks.

Lack of TLK function leads to severe chromatin defects in flies and worms ${ }^{37,38}$, and TLK mutations jeopardize gene silencing in plants $^{54}$. Our work proposes that TLK signalling ensures efficient histone delivery during genome duplication, hereby facilitating proper restoration of the epigenetic landscape. An exciting perspective for future research is that TLKs might target additional factors involved in chromatin replication and coordinate a cellular response to histone demand.

\footnotetext{
Methods

Cloning. Plasmids used in this study are listed in Supplementary Table 1. Human Asf1 was inserted in frame into pcDNA5/FRT/TO-FLAG-HA ${ }^{51}$. QuickChange Site-Directed Mutagenesis (Stratagene) was used to generate Asfla mutants $4 \mathrm{D}$ (S166D, S175D, S192D, S199D), 4A (S166A, S175A, S192A, S199A), 3A (S166A, S175A, S192A), 2A (S166A, S175A), and Asf1b mutants 2E (S169E, S198E), Asf1b
} 2A (S169A, S198A), 1A (S198A). All constructs were verified by sequencing.
Cell culture and transfections. Cell lines are listed in Supplementary Table 2. U-2-OS and Hela S3 cell lines were grown in DMEM (Gibco) supplemented with penicillin/streptomycin, 10\% FBS (Hyclone) and drugs for selection (see Supplementary Table 2). HeLa S3 cells stably expressing OneStrep-tagged Asf1 ( $a$ and $b$ ) were described ${ }^{19,51}$. Asf1-inducible cell lines were generated in U-2-OS Flp-In T-Rex (Invitrogen) as single-cell clones. TLK1-inducible cell lines were generated as described ${ }^{35}$. For expression of FLAG-HA-Asfla (e-Asfla) wild-type (wt) and mutants, cells were induced with $1 \mu \mathrm{g} \mathrm{ml}^{-1}$ tetracycline for 8 to $14 \mathrm{~h}$. For expression of GFP-TLK1, cells were induced for $24 \mathrm{~h}$. Expression plasmids were introduced by FuGENE 6 (Promega) transfection or by electroporation using a GenePulser Xcell (BIO-RAD). Cells were transfected with siRNAs at $100 \mathrm{nM}$ concentration using Oligofectamine (Invitrogen). The Asf1 ( $a$ and b) targeting siRNAs were described ${ }^{19}$, siRNAs against FLASH (sense strand $5^{\prime}$-CCGCAAG GAUGAAGAAAUAdTdT-3') and SLBP (sense strand 5'-GGAUGUGAUU UGCAAGAAAdTdT- $3^{\prime}$ ) were from Sigma. DNA content was analysed by propidium iodide staining on a FACScalibur (BD) using CellQuest Pro 6.0 software (BD). Cell cycle profiles were generated by FlowJo 8.8.4 software (Tree Star) and quantified by Dean-Jett-Fox model and manual gating. Cells were synchronized by a single thymidine (Sigma, $2 \mathrm{mM}$ ) block for $17 \mathrm{~h}$ (Fig. 3e) or hydroxyurea (Sigma, $3 \mathrm{mM}$ ) for $20 \mathrm{~h}$ (Fig. 3d) and released into fresh media with deoxycytidine (Sigma, $24 \mu \mathrm{M})$. Where indicated, cells were treated with $\mathrm{HU}(3 \mathrm{mM}, 1 \mathrm{~h}), \mathrm{UCN}-01$ ( $300 \mathrm{nM}, 1 \mathrm{~h}$ ) or CHX $\left(50 \mu \mathrm{g} \mathrm{ml}^{-1}\right.$, from 1 to $4 \mathrm{~h}$; all from Sigma). UCN-01 was added $15 \mathrm{~min}$ before $\mathrm{HU}$.

Complex purification and gel-filtration. Purification of OneStrep-Asfla/b complexes for mass spectrometry analysis from cytosolic and nuclear extracts was performed essentially as described ${ }^{11,51}$ using high-stringency washes (see below). Cytosolic and nuclear extracts for isolation of the e-Asfla complexes were prepared as described ${ }^{11}$. In brief, cells were washed with ice-cold PBS, swollen in buffer E (20 mM Hepes- $\mathrm{KOH}$ pH 7.8, $5 \mathrm{mM}$ potassium acetate, $0.5 \mathrm{mM} \mathrm{MgCl}$ ) supplemented with inhibitors ( $1 \mathrm{mM}$ DTT, $10 \mu \mathrm{g} \mathrm{ml}^{-1}$ leupeptin, $10 \mu \mathrm{g} \mathrm{ml}{ }^{-1}$ pepstatin, $0.1 \mathrm{mM}$ PMSF, $0.2 \mathrm{mM}$ sodium vanadate, $5 \mathrm{mM}$ sodium fluoride, $10 \mathrm{mM}$ beta-glycerolphosphate). Cells were homogenized using loose type pestle. Pelleted nuclei $(1,500 \mathrm{~g}, 5 \mathrm{~min})$ were extracted with $2.5 \times$ volume of extraction buffer $\mathrm{N}$ (buffer E with inhibitors, $540 \mathrm{mM} \mathrm{NaCl}, 10 \%$ glycerol). Extracts were pre-cleared with agarose beads (SuperFlow 6, IBA) for $1 \mathrm{~h}$, followed by $2 \mathrm{~h}$ incubation with antiFLAG M2-agarose beads (Sigma). We used low-stringency washes for isolation of nuclear complexes (Buffer A: $150 \mathrm{mM} \mathrm{NaCl}, 50 \mathrm{mM}$ Tris pH 7.8, $0.2 \mathrm{mM}$ EDTA, $0.2 \%$ NP- $40,1 \mathrm{mM} \mathrm{DTT}, 5 \%$ glycerol, protease and phosphatase inhibitors) and high-stringency washes (buffer $\mathrm{A}$, containing $500 \mathrm{mM} \mathrm{NaCl}$ ) for purification of cytosolic complexes. Complexes were eluted from the beads with a FLAG-peptide $\left(400 \mu \mathrm{g} \mathrm{ml}^{-1}\right)$. FLAG-HA-Asflb complexes were isolated under low stringency using anti-FLAG beads from whole-cell extracts $(300 \mathrm{mM} \mathrm{NaCl}, 0.5 \% \mathrm{NP}-40$, $50 \mathrm{mM}$ Tris $\mathrm{pH}$ 7.5, 0.2 mM EDTA, 5\% glycerol and inhibitors). For fractionation of cells by triton extraction (Fig. $6 c-e$ ), soluble proteins were extracted in CSK-T ( $10 \mathrm{mM}$ PIPES $\mathrm{pH} 7,100 \mathrm{mM} \mathrm{NaCl}, 300 \mathrm{mM}$ sucrose, $3 \mathrm{mM}$ $\mathrm{MgCl} 2,0.5 \%$ Triton, inhibitors) for $8 \mathrm{~min}$ before the pellet was isolated and washed once in CSK-T. Total cell lysates (Figs 1f,2a,3a) were made in Laemmli sample buffer (LSB: $50 \mathrm{mM}$ Tris pH 6.8, $100 \mathrm{mM}$ DTT, $2 \%$ SDS, 7.5\% glycerol, bromphenol blue) and treated with Benzonase (Novagen) for $30 \mathrm{~min}$ at room temperature.

Gel filtration analysis of mid-S phase cytosolic extract from inducible U-2-OS cells expressing similar levels e-Asfla wt/4D/4A was performed as described ${ }^{6,51}$ with minor modifications. In brief, $1 \mathrm{mg}$ of extracts was loaded on SuperdexG200 10/300 GL column (GE Healthcare) and complexes were eluted in buffer E containing $100 \mathrm{mM} \mathrm{NaCl}, 5 \%$ glycerol and inhibitors at a flow of $0.3 \mathrm{ml} \mathrm{min}^{-1}$ in $400 \mu \mathrm{l}$ fractions. Proteins were precipitated by trichloroacetic acid and $\mathrm{Na}$ deoxycholate and analysed by western blotting with Gel Filtration Calibration Kit HMW (high molecular weight, GE Healthcare) as a size marker.

Antibodies. Asf1a pS166 rabbit polyclonal antibodies were generated against Asfla phospho-peptide (CTEKLEDAESpSNPNL) and double affinity purified (Genscript). TLK1 ('TALAF') rabbit polyclonal antibodies were generated against the peptide KKKSPTALAFGDHPIVQPKQLSFK. Primary antibodies and dilutions are listed in Supplementary Table 3.

Secondary antibodies (anti-mouse (115-035-068, 1:20,000), anti-rabbit (711-035-152, 1:20,000) and anti-rat (122-035-068, 1:20,000)) conjugated with HRP were from Jackson ImmunoResearch Labs; anti-rabbit-Alexa488 (1:1,000) and anti-mouse-Alexa568 (1:1,000) were from Invitrogen.

Full scans of all western blots are included in Supplementary Figs 8-10. For quantification of western blots, films were scanned and signal intensities were analysed with NIH Image J 1.41.

In vitro kinase reactions. Recombinant myc-TLK1 wild-type (wt) or kinase-inactive (ki) were purified from Sf9 insect cells infected with recombinant baculovirus ${ }^{34}$, using protein $\mathrm{G}$ sepharose beads (GE Healthcare) coupled to myc antibodies. Myc-TLK1 was eluted in low pH buffer (100 mM glycin pH 2.5, $1 \mathrm{mM}$ DTT, $2.5 \mathrm{mM} \mathrm{NaF}$ ), and $\mathrm{pH}$ was adjusted by addition of $1 \mathrm{M}$ Tris $\mathrm{pH} 8.0$ (supplemented with $1 \mathrm{mM}$ DTT, $2.5 \mathrm{mM} \mathrm{NaF}$ ). In vitro TLK1 kinase assays were performed essentially as described ${ }^{34,35}$. In brief, myc-TLK1 was incubated in $25 \mu \mathrm{l}$ kinase assay buffer (KAB - $50 \mathrm{mM}$ HEPES pH 7.2, $10 \mathrm{mM} \mathrm{MgCl}_{2}, 5 \mathrm{mM} \mathrm{MnCl}_{2}$,

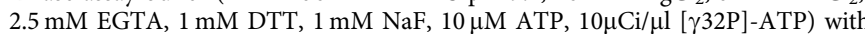


$1 \mu \mathrm{g}$ GST-Asfla for $30 \mathrm{~min}$ and $30^{\circ} \mathrm{C}$. Kinase reactions were stopped by the addition of $4 \mathrm{xLSB}$, and reaction products were resolved on a $12 \%$ SDS-PAGE. $\left[{ }^{32} \mathrm{P}\right]$ incorporation was quantified on a PhosphorImager FLA3000 (Fujifilm, Tokyo). For identification of TLK1 phospho-sites in Asfl (a and b) by mass spectrometry, the kinase reaction contained $50 \mu \mathrm{M}$ ATP, $6 \mu \mathrm{g}$ of recombinant Asfl (a or b) and myc-TLK1 $(2 \mu \mathrm{g})$. Reaction products were resolved on a 15\% SDS-PAGE gel and stained with Coomassie Blue. Slow-migrating bands were cut and analysed by LC-MS/MS.

Protein purification and pull-down assays. GST-Asf1 fusion proteins were expressed in E. coli and purified on glutathione-Sepharose beads 4B (GE Healthcare Life Science) using buffer containing $20 \mathrm{mM}$ Tris- $\mathrm{HCl} \mathrm{pH} \mathrm{8,500} \mathrm{mM} \mathrm{NaCl}$, $0.1 \%$ Triton X100, $0.5 \mathrm{mg} \mathrm{ml}^{-1}$ Lysosyme and protease inhibitors ${ }^{32}$. Proteins were eluted from the glutathione-Sepharose beads with $20 \mathrm{mM}$ Gluthathion (GE Healthcare Life Science) in elution buffer $(100 \mathrm{mM}$ Tris-HCl pH 8, $120 \mathrm{mM} \mathrm{NaCl}$, $0.1 \%$ Triton X100, $10 \%$ Glycerol and proteinase inhibitors). Where indicated, Asf1 was cleaved from GST with PreScission protease (GE Healthcare).

For in vitro binding, myc-TLK1 wt or ki on anti-myc sepharose beads were incubated with Asfla proteins for $2 \mathrm{~h}$ at $4{ }^{\circ} \mathrm{C}$ in buffer A. The reactions were washed five times with buffer A, and bound proteins were solubilized in Laemmli sample buffer (4xLSB: $200 \mathrm{mM}$ Tris pH 6.8, $400 \mathrm{mM}$ DTT, 8\% SDS, 30\% glycerol, bromphenol blue).

For GST pull-downs, GST-Asfla proteins were coupled to glutathionesepharose beads (GE Healthcare) for $1 \mathrm{~h}$ at $4{ }^{\circ} \mathrm{C}$ and incubated with cell extracts in buffer $\mathrm{A}$ for $3 \mathrm{~h}$ at $4^{\circ} \mathrm{C}$. Reactions were washed six times with buffer $\mathrm{A}$ and bound proteins were solubilized in LSB. Where indicated, GST-Asfla was phosphorylated by myc-TLK1 wt or ki before pull-down reactions. After the kinase reaction, GST proteins were separated from myc-TLK1-beads by centrifugation.

Mass spectrometric analyses of Asf1 phosphorylation sites. Asfla and Asflb gel bands were subjected to in-gel digestion as previously described ${ }^{55}$, with replicate bands digested with either $100 \mathrm{ng}$ of trypsin (overnight at $37^{\circ} \mathrm{C}$ ) or thermolysin (Calbiochem, $60 \mathrm{~min}$ at $56^{\circ} \mathrm{C}$ ). Phosphopeptide enrichment was performed on titanium dioxide microcolumns before the resulting eluates were desalted with Poros R3 material ${ }^{56,57}$. LC-MS/MS was conducted on an EASY-nLC system (Thermo Fisher Scientific) coupled to an LTQ-Orbitrap XL mass spectrometer (Thermo Fisher Scientific) as reported ${ }^{58}$, with a minimum threshold of 30,000 precursor ions required to trigger MS/MS spectrum acquisition. Using Proteome Discoverer 1.0 software (Thermo Fisher Scientific), raw data files were submitted to Mascot database searches 2.2.04 (Matrix Science). An NCBI non-redundant database (20090412) containing 222,460 human protein sequences was searched with the following parameters: ESI-TRAP was selected as the instrument type, with specified mass tolerances of 10 p.p.m. (precursor) and $0.6 \mathrm{Da}$ (fragment). $\mathrm{N}$-terminal protein acetylation, methionine oxidation and phosphorylation (serine, threonine and tyrosine) were set as variable modifications. Cysteine carbamidomethylation was included as a fixed modification. Thermolytic searches were permitted up to nine missed cleavages, with cleavage specificity set $\mathrm{N}$-terminal to valine, isoleucine, leucine, phenylalanine, alanine and methionine. Tryptic searches were conducted with one missed cleavage permissible. Only Asfla and Asflb phosphopeptides with Mascot ion scores above 20 were considered for evaluation, which involved manual validation of the MS/MS spectrum to determine the site of phosphorylation. The Asf1 phosphopeptide mass spectrometry data have been deposited to the ProteomeXchange Consortium (http://www.proteomexchange.org) via the PRIDE partner repository ${ }^{59}$ with the data set identifier PXD000686.

SILAC-based proteomics. For SILAC (Stable Isotope Labelling with Amino acids in Cell culture), conditional cell lines expressing e-Asfiā wt and phospho-mimetic $(4 \bar{D})$ mutants were grown in medium depleted for Arginine and Lysine (Thermo Scientific, RPMI 1640) and supplemented with dialysed FBS (Invitrogen, 26400036), MEM non-essential amino acids (Invitrogen, 11140) and Glutamax (Invitrogen, 35050-038). SILAC medium for e-Asfla wt cells was complemented with $66 \mathrm{mgl}^{-1}$ of arginine and $100 \mathrm{mgl}^{-1}$ of lysine (light, Arg0/Lys0, Sigma), while medium for e-Asf1 4D cell lines was complemented with heavy lysine and arginine (Arg6/Lys8, Cambridge lab, CNLM-291-0.25, CLM-2265-0). Cells were maintained for 8 to 9 divisions in heavy or light medium before analysis. e-Asfla wt and 4D were expressed at a similar level and complexes were isolated under low-stringency conditions (buffer A) from whole-cell extracts. Before elution from the FLAG M2 beads, e-Asfla wt and $4 \mathrm{D}$ complexes were mixed 1:1. Eluted proteins were run on SDS-PAGE, excised from the gel and subjected to LC-MS/MS. In-gel digestion was performed as described ${ }^{55}$, except chloroacetamide was used in place of iodoacetamide as the alkylating agent ${ }^{60}$. LC-MS/MS was executed with an EASYnLC system coupled to an Q Exactive mass spectrometer (Thermo Fisher Scientific) using the 76 min 'sensitive' method as previously described ${ }^{61}$ with a $2 \mathrm{Da}$ isolation width. Data files (technical duplicates) were processed with MaxQuant 1.2.7.1, with searches conducted against a UniProt database containing 86875 human protein sequences (2012_06) concatenated with reverse entries and a list of protein sequences from commonly observed contaminants. The enzyme specificity was set to trypsin/P with a maximum of two miscleavages allowed. Recalibrated spectra were searched with a 6 p.p.m. MS tolerance and 20 p.p.m. MS/MS tolerance.
A false discovery rate of $1 \%$ was employed for protein and peptide identifications, with a minimum of two peptides required for each protein identification. Criteria for peptide identification included a minimum score of 60 and a minimum peptide length of six amino acids. For protein quantification, a minimum of two ratio counts was required.

Immunostaining. Cells were either pre-extracted with $0.5 \%$ Triton in CSK buffer (10 mM PIPES pH 7, $100 \mathrm{mM} \mathrm{NaCl}, 300 \mathrm{mM}$ sucrose, $3 \mathrm{mM} \mathrm{MgCl} 2)$ for $5 \mathrm{~min}$ at $4{ }^{\circ} \mathrm{C}$ to remove soluble proteins or fixed directly with $4 \%$ formaldehyde $(10 \mathrm{~min}$ at room temperature) and permeabilized with $0.2 \%$ Triton. To reveal the epitope recognized by the PCNA antibody, cells were treated with methanol for 20 min at $-20^{\circ} \mathrm{C}$. EdU labelling $(40 \mu \mathrm{M}, 20 \mathrm{~min})$ was detected by Click-iT Cell Reaction (Invitrogen). Fluorescence images were collected on a DeltaVision system and analysed by SoftWoRX 5.0.0 or on a Zeiss inverted microscope Axiovert $200 \mathrm{M}$ equipped with LSM510 Laser module with a 63x objective (C-APOCHROMAT, water immersion, $63-/ 1.2 \mathrm{~W}$ Korr). Brightness and contrast were adjusted using Adobe Photoshop CS5 (12.0.4).

Surface plasmon resonance. Human recombinant H3.1-H4 tetramers (NEB, M2509S) were diluted 200-fold to $50 \mathrm{nM}$ in running buffer (10 mM Tris $\mathrm{pH} 7.8$, $100 \mathrm{mM} \mathrm{NaCl}, 50 \mu \mathrm{M}$ EDTA, $0.5 \mathrm{mM}$ DTT and $0.05 \%(\mathrm{v} / \mathrm{v}) \mathrm{P}-20)$ to promote the dissociation into $\mathrm{H} 3.1-\mathrm{H} 4$ heterodimers ${ }^{62}$. H3.1- $\mathrm{H} 4$ was non-covalently captured by electrostatic interaction directly onto one of the flow cells in a C1 Sensor Chip (GE Healthcare) by a $225 \mathrm{~s}$ injection at $10 \mu \mathrm{lmin}^{-1}$ followed by a $17 \mathrm{~min}$ washing step with running buffer at $20 \mu \mathrm{min}^{-1}$ to attenuate further decay of the captured surface during the subsequent kinetic experiment. Monomeric status of recombinant Asfla (cleaved from GST-tag) was confirmed by size exclusion chromatography (SuperdexG200 5/150 GL). Next, Asfla preparations (1-73 nM dilutions in running buffer) were allowed to interact with the H3.1-H4 captured on the surface and with matched un-captured control surface for $200 \mathrm{~s}$ before the dissociation phase was recorded for $45 \mathrm{~min}$. The surface was regenerated by two consecutive $30 \mathrm{~s}$ injections of $0.3 \%(\mathrm{w} / \mathrm{v})$ SDS. All measurements were performed with a flow rate of $20 \mu \mathrm{min}^{-1}$ at $20^{\circ} \mathrm{C}$ using a BiaCoreT200 (GE Healthcare). The recorded data were processed by double referencing using the paired buffer runs analysed immediately before the sample injection. Kinetic rate constants for the interactions were derived by local fitting double referenced data to a simple bimolecular interaction model using the BiaEvaluation 4.1 software.

Statistical analysis. Data were subjected to a two-tailed Student's $t$-test to resolve statistical significance (Prism 5.0). Results from at least three independent experiments are presented and error bars indicate s.d.

\section{References}

1. Alabert, C. \& Groth, A. Chromatin replication and epigenome maintenance. Nat. Rev. Mol. Cell. Biol. 13, 153-167 (2012).

2. Margueron, R. \& Reinberg, D. Chromatin structure and the inheritance of epigenetic information. Nat. Rev. Genet. 11, 285-296 (2010).

3. Probst, A. V., Dunleavy, E. \& Almouzni, G. Epigenetic inheritance during the cell cycle. Nat. Rev. Mol. Cell. Biol. 10, 192-206 (2009).

4. Marzluff, W. F., Wagner, E. J. \& Duronio, R. J. Metabolism and regulation of canonical histone mRNAs: life without a poly(A) tail. Nat. Rev. Genet. 9, 843-854 (2008).

5. Tyler, J. K. et al. The RCAF complex mediates chromatin assembly during DNA replication and repair. Nature 402, 555-560 (1999).

6. Groth, A. et al. Human Asf1 Regulates the Flow of S Phase Histones during Replicational Stress. Mol. Cell. 17, 301-311 (2005).

7. Schulz, L. L. \& Tyler, J. K. The histone chaperone ASF1 localizes to active DNA replication forks to mediate efficient DNA replication. FASEB J. 20, 488-490 (2006).

8. Grigsby, I. F., Rutledge, E. M., Morton, C. A. \& Finger, F. P. Functional redundancy of two C. elegans homologs of the histone chaperone Asf1 in germline DNA replication. Dev. Biol. 329, 64-79 (2009).

9. Umehara, T., Chimura, T., Ichikawa, N. \& Horikoshi, M. Polyanionic stretchdeleted histone chaperone cia1/Asflp is functional both in vivo and in vitro. Genes Cells 7, 59-73 (2002).

10. Campos, E. I. et al. The program for processing newly synthesized histones H3.1 and H4. Nat. Struct. Mol. Biol. 17, 1343-1351 (2010).

11. Jasencakova, Z. et al. Replication stress interferes with histone recycling and predeposition marking of new histones. Mol. Cell. 37, 736-743 (2010).

12. Alvarez, F. et al. Sequential establishment of marks on soluble histones $h 3$ and h4. J. Biol. Chem. 286, 17714-17721 (2011).

13. Cook, A. J., Gurard-Levin, Z. A., Vassias, I. \& Almouzni, G. A specific function for the histone chaperone NASP to fine-tune a reservoir of soluble $\mathrm{H} 3-\mathrm{H} 4$ in the histone supply chain. Mol. Cell. 44, 918-927 (2011).

14. Tyler, J. K. et al. Interaction between the Drosophila CAF-1 and ASF1 chromatin assembly factors. Mol. Cell. Biol. 21, 6574-6584 (2001).

15. Mello, J. A. et al. Human Asf1 and CAF-1 interact and synergize in a repair-coupled nucleosome assembly pathway. EMBO Rep. 3, 329-334 (2002). 
16. Winkler, D. D., Zhou, H., Dar, M. A., Zhang, Z. \& Luger, K. Yeast CAF-1 assembles histone (H3-H4)2 tetramers prior to DNA deposition. Nucleic Acids Res. 40, 10139-10149 (2012).

17. Shibahara, K. \& Stillman, B. Replication-dependent marking of DNA by PCNA facilitates CAF-1-coupled inheritance of chromatin. Cell 96, 575-585 (1999).

18. Smith, S. \& Stillman, B. Stepwise assembly of chromatin during DNA replication in vitro. EMBO J. 10, 971-980 (1991).

19. Groth, A. et al. Regulation of replication fork progression through histone supply and demand. Science 318, 1928-1931 (2007).

20. Tagami, H., Ray-Gallet, D., Almouzni, G. \& Nakatani, Y. Histone H3.1 and H3.3 complexes mediate nucleosome assembly pathways dependent or independent of DNA synthesis. Cell 116, 51-61 (2004).

21. Tang, Y. et al. Structure of a human ASF1a-HIRA complex and insights into specificity of histone chaperone complex assembly. Nat. Struct. Mol. Biol. 13, 921-929 (2006).

22. Corpet, A. et al. Asflb, the necessary Asf1 isoform for proliferation, is predictive of outcome in breast cancer. EMBO J. 30, 480-493 (2011).

23. Zhang, R. et al. Formation of MacroH2A-containing senescence-associated heterochromatin foci and senescence driven by ASF1a and HIRA. Dev. Cell 8, 19-30 (2005).

24. Hayashi, R. et al. Transcriptional regulation of human chromatin assembly factor ASF1. DNA. Cell. Biol. 26, 91-99 (2007).

25. Mousson, F. et al. Structural basis for the interaction of Asfl with histone $\mathrm{H} 3$ and its functional implications. Proc. Natl Acad. Sci. USA 102, 5975-5980 (2005).

26. Daganzo, S. M. et al. Structure and function of the conserved core of histone deposition protein Asf1. Curr. Biol. 13, 2148-2158 (2003).

27. English, C. M., Adkins, M. W., Carson, J. J., Churchill, M. E. \& Tyler, J. K. Structural basis for the histone chaperone activity of Asf1. Cell 127, 495-508 (2006).

28. Natsume, R. et al. Structure and function of the histone chaperone CIA/ASF1 complexed with histones H3 and H4. Nature 446, 338-341 (2007).

29. Liu, W. H., Roemer, S. C., Port, A. M. \& Churchill, M. E. CAF-1-induced oligomerization of histones $\mathrm{H} 3 / \mathrm{H} 4$ and mutually exclusive interactions with Asf1 guide $\mathrm{H} 3 / \mathrm{H} 4$ transitions among histone chaperones and DNA. Nucleic Acids Res. 40, 11229-11239 (2012).

30. Zhang, W. et al. Structural plasticity of histones H3-H4 facilitates their allosteric exchange between RbAp48 and ASF1. Nat. Struct. Mol. Biol. 20, 29-35 (2013).

31. Dennehey, B. K. et al. The C terminus of the histone chaperone Asf1 cross-links to histone $\mathrm{H} 3$ in yeast and promotes interaction with histones $\mathrm{H} 3$ and $\mathrm{H} 4$. Mol. Cell. Biol. 33, 605-621 (2013)

32. Sillje, H. H. \& Nigg, E. A. Identification of human Asf1 chromatin assembly factors as substrates of Tousled-like kinases. Curr. Biol. 11, 1068-1073 (2001).

33. Mousson, F., Ochsenbein, F. \& Mann, C. The histone chaperone Asfl at the crossroads of chromatin and DNA checkpoint pathways. Chromosoma 116, 79-93 (2007).

34. Sillje, H. H., Takahashi, K., Tanaka, K., Van Houwe, G. \& Nigg, E. A. Mammalian homologues of the plant Tousled gene code for cell-cycle-regulated kinases with maximal activities linked to ongoing DNA replication. EMBO J. 18, 5691-5702 (1999).

35. Groth, A. et al. Human Tousled like kinases are targeted by an ATM- and Chk1-dependent DNA damage checkpoint. EMBO J. 22, 1676-1687 (2003).

36. Krause, D. R. et al. Suppression of Tousled-like kinase activity after DNA damage or replication block requires ATM, NBS1 and Chk1. Oncogene 22, 5927-5937 (2003).

37. Carrera, P. et al. Tousled-like kinase functions with the chromatin assembly pathway regulating nuclear divisions. Genes Dev. 17, 2578-2590 (2003).

38. Han, Z., Saam, J. R., Adams, H. P., Mango, S. E. \& Schumacher, J. M. The C. elegans Tousled-like kinase (TLK-1) has an essential role in transcription. Curr. Biol. 13, 1921-1929 (2003).

39. Hashimoto, M., Matsui, T., Iwabuchi, K. \& Date, T. PKU-beta/TLK1 regulates myosin II activities, and is required for accurate equaled chromosome segregation. Mutat. Res. 657, 63-67 (2008).

40. Pilyugin, M., Demmers, J., Verrijzer, C. P., Karch, F. \& Moshkin, Y. M. Phosphorylation-mediated control of histone chaperone ASF1 levels by Tousled-like kinases. PLoS One 4, e8328 (2009).

41. Seale, R. L. \& Simpson, R. T. Effects of cycloheximide on chromatin biosynthesis. J. Mol. Biol. 94, 479-501 (1975).

42. Weintraub, H. A possible role for histone in the synthesis of DNA. Nature 240, 449-453 (1972)

43. Zhao, X., McKillop-Smith, S. \& Muller, B. The human histone gene expression regulator HBP/SLBP is required for histone and DNA synthesis, cell cycle progression and cell proliferation in mitotic cells. J. Cell. Sci. 117, 6043-6051 (2004).

44. Barcaroli, D. et al. FLASH is required for histone transcription and S-phase progression. Proc. Natl Acad. Sci. USA 103, 14808-14812 (2006).

45. Olsen, J. V. et al. Quantitative phosphoproteomics reveals widespread full phosphorylation site occupancy during mitosis. Sci. Signal. 3, ra3 (2010).

46. Hsu, P. P. et al. The mTOR-regulated phosphoproteome reveals a mechanism of mTORC1-mediated inhibition of growth factor signaling. Science 332, 1317-1322 (2011)
47. Tamburini, B. A., Carson, J. J., Linger, J. G. \& Tyler, J. K. Dominant mutants of the Saccharomyces cerevisiae ASF1 histone chaperone bypass the need for CAF-1 in transcriptional silencing by altering histone and Sir protein recruitment. Genetics 173, 599-610 (2006).

48. Ahmad, K. \& Henikoff, S. The histone variant H3.3 marks active chromatin by replication-independent nucleosome assembly. Mol. Cell. 9, 1191-1200 (2002)

49. Smith, S. \& Stillman, B. Purification and characterization of CAF-I, a human cell factor required for chromatin assembly during DNA replication in vitro. Cell 58, 15-25 (1989).

50. Ray-Gallet, D. et al. Dynamics of histone $\mathrm{H} 3$ deposition in vivo reveal a nucleosome gap-filling mechanism for $\mathrm{H} 3.3$ to maintain chromatin integrity. Mol. Cell. 44, 928-941 (2011).

51. Ask, K. et al. Codanin-1, mutated in the anaemic disease CDAI, regulates Asf1 function in S-phase histone supply. EMBO J. 31, 2013-2023 (2012).

52. De Benedetti, A. Tousled kinase TLK1B counteracts the effect of Asf1 in inhibition of histone H3-H4 tetramer formation. BMC Res. Notes 2, 128 (2009).

53. Jiao, Y. et al. Surprising complexity of the Asf1 histone chaperone-Rad53 kinase interaction. Proc. Natl Acad. Sci. USA 109, 2866-2871 (2012).

54. Wang, Y. et al. The protein kinase TOUSLED is required for maintenance of transcriptional gene silencing in Arabidopsis. EMBO Rep. 8, 77-83 (2007).

55. Shevchenko, A. et al. Linking genome and proteome by mass spectrometry: large-scale identification of yeast proteins from two dimensional gels. Proc. Natl Acad. Sci. USA 93, 14440-14445 (1996).

56. Larsen, M. R., Thingholm, T. E., Jensen, O. N., Roepstorff, P. \& Jorgensen, T. J. Highly selective enrichment of phosphorylated peptides from peptide mixtures using titanium dioxide microcolumns. Mol. Cell. Proteomics 4, 873-886 (2005).

57. Thingholm, T. E., Jorgensen, T. J., Jensen, O. N. \& Larsen, M. R. Highly selective enrichment of phosphorylated peptides using titanium dioxide. Nat. Protoc. 1, 1929-1935 (2006).

58. Zhao, X. et al. Phosphoproteome analysis of functional mitochondria isolated from resting human muscle reveals extensive phosphorylation of inner membrane protein complexes and enzymes. Mol. Cell. Proteomics 10, 000299 (2011).

59. Vizcaino, J. A. et al. The PRoteomics IDEntifications (PRIDE) database and associated tools: status in 2013. Nucleic Acids Res. 41, D1063-D1069 (2013).

60. Nielsen, M. L. et al. Iodoacetamide-induced artifact mimics ubiquitination in mass spectrometry. Nat. Methods 5, 459-460 (2008).

61. Kelstrup, C. D., Young, C., Lavallee, R., Nielsen, M. L. \& Olsen, J. V. Optimized fast and sensitive acquisition methods for shotgun proteomics on a quadrupole orbitrap mass spectrometer. J. Proteome Res. (2012).

62. Winkler, D. D., Zhou, H., Dar, M. A., Zhang, Z. \& Luger, K. Yeast CAF-1 assembles histone (H3-H4) 2 tetramers prior to DNA deposition. Nucleic Acids Res. 40, 10139-10149 (2012).

\section{Acknowledgements}

We thank K. Hansen for fruitful discussions and critical reading of the manuscript. This work was supported by a generous grant from the Danish National Research Foundation to the Center for Epigenetics (DNRF82; C.Y., O.N.J. and A.G.). A.G. is an EMBO Young Investigator and her laboratory is supported by a European Research Council Starting Grant (ERC2011StG), the Lundbeck Foundation, the Danish Cancer Society and the Danish Medical Research Council, the Novo Nordisk Foundation and FP7 Marie Curie Actions ITN Nucleosome4D and aDDRess.

\section{Author contributions}

I.M.K. designed and performed the majority of the experiments and analysed the data. C.Y. and O.N.J. designed experiments for analysis of protein phosphorylation by mass spectrometry. C.Y. performed the mass spectrometry experiments and data analysis. C.B.S. performed experiments on Asflb phosphorylation, complementation, TLK localization and chromatin-binding. P.M. generated Asfl expression vectors and mutants. Z.J. and K.A. isolated Asfl complexes for in vivo phospho-site mapping. J.M. contributed to the histone depletion experiments. M.P. measured affinities between Asf1 and histones using surface plasmon resonance. A.G. supervised the project and conceived the strategy. I.M.K. and A.G. wrote the manuscript. C.B.S., Z.J., C.Y., M.P., O.N.J. and M.L.N. commented on the manuscript.

\section{Additional information}

Accession codes: The Asfl phosphopeptide mass spectrometry data is available from the ProteomeXchange Consortium under the data set identifier PXD000686.

Supplementary Information accompanies this paper at http://www.nature.com/ naturecommunications

Competing financial interests: The authors declare no competing financial interests.

Reprints and permission information is available online at http://npg.nature.com/ reprintsandpermissions/

How to cite this article: Klimovskaya, I. M. et al. Tousled-like kinases phosphorylate Asf1 to promote histone supply during DNA replication. Nat. Commun. 5:3394 doi: $10.1038 /$ ncomms4394 (2014). 